\title{
Modified end-stage renal disease risk score for Chinese type 2 diabetic patients - the Hong Kong Diabetes Registry
}

\author{
X. L. Yang • W. Y. So • A. P. S. Kong • C. S. Ho • \\ C. W. K. Lam • M. H. L. Ng • R. R. Lyu • D. D. Yin • \\ C. C. Chow • C. S. Cockram • P. C. Y. Tong • \\ J. C. N. Chan
}

Received: 15 October 2006 / Accepted: 22 January 2007 / Published online: 13 April 2007

(C) Springer-Verlag 2007

To the Editor: Ma et al. [1] recalibrated the Modification of Diet in Renal Disease (MDRD) equation for estimation of the GFR of Chinese individuals. It was reported that it is necessary for the GFR derived from the original MDRD equation to be multiplied by an adjusting coefficient of 1.233. This recalibration for Chinese individuals may have a significant effect on the end-stage renal disease (ESRD) risk score [2]. If a GFR of $15 \mathrm{ml} \mathrm{min}^{-1} 1.73 \mathrm{~m}^{-2}$ is used to define ESRD, patients with values between 12.17 and $15.00 \mathrm{ml} \mathrm{min}{ }^{-1} 1.73 \mathrm{~m}^{-2}$ estimated by the original MDRD

Electronic supplementary material The online version of this article (doi:10.1007/s00125-007-0639-7) contains supplementary material, which is available to authorised users.

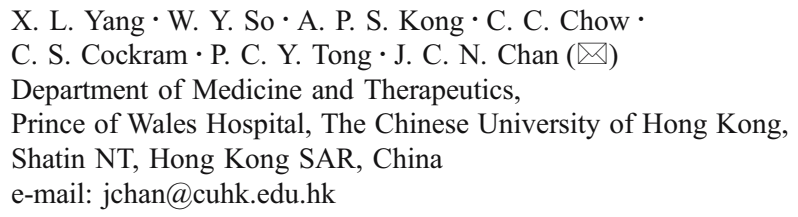

\section{H. L. Ng}

Department of Anatomical and Cellular Pathology,

The Chinese University of Hong Kong,

Shatin NT, Hong Kong SAR, China

A. P. S. Kong

Li Ka Shing Institute of Health Sciences

\& Department of Medicine and Therapeutics,

The Chinese University of Hong Kong,

Shatin NT, Hong Kong SAR, China

R. R. Lyu • D. D. Yin

Worldwide Outcomes Research, Merck \& Co.,

Whitehouse Station, NJ, USA equation will no longer meet the definition of ESRD by the Chinese recalibrated MDRD (C-MDRD) equation. The aim of the present study was to examine the effect of the C-MDRD equation on the validity of the ESRD risk score for Chinese patients with type 2 diabetes mellitus [2].

The study population, clinical measurements, definition of ESRD and laboratory assays have been described previously [2]. Due to the reduced number of patients with ESRD following application of the C-MDRD equation, the updated database of the Hong Kong Diabetes Registry, which contains 4,799 previously recruited patients [2] and 3,121 patients not previously reported, was used to examine the impact of the CMDRD equation on the ESRD risk score. The C-MDRD [1] used to estimate GFR in $\mathrm{ml} \mathrm{min}^{-1} 1.73 \mathrm{~m}^{-2}$ was: $\mathrm{GFR}=186 \times$ $(\mathrm{SCR} \times 0.011)^{-1.154} \times(\mathrm{age})^{-0.203} \times(0.742$ if female $) \times 1.233$, where SCR is serum creatinine expressed as $\mu \mathrm{mol} / 1$ (original $\mathrm{mg} / \mathrm{dl}$ converted into $\mu \mathrm{mol} / \mathrm{l}$ ) and 1.233 is the adjusting coefficient.

As previously described, ESRD was defined as: (1) death due to diabetes with renal manifestations or renal failure (the International Classification of Diseases, 9th Revision [ICD-9] code 250.4, 585, 586); (2) hospitalisation due to non-fatal renal failure (ICD-9 codes 585 or 586); (3) GFR $<15 \mathrm{ml} \mathrm{min}^{-1} 1.73 \mathrm{~m}^{-2}$ [2]. Follow-up time was calculated as the period from enrolment up to either the date of occurrence of any one of the above events during followup or 30 July 2005, whichever came first. Of 7,920 patients, 7,516 type 2 diabetic patients who did not have ESRD (GFR $\geq 15 \mathrm{ml} \mathrm{min}^{-1} 1.73 \mathrm{~m}^{-2}$ or hospitalisation due to renal failure) at enrolment were analysed in the study. A total of 40,171.6 person-years were observed. At enrolment, $10.2 \%(n=765)$ of the 7, 516 patients had a GFR of between 15 and $60 \mathrm{ml} \mathrm{min}{ }^{-1} 1.73 \mathrm{~m}^{-2}$, and $15.54 \%(n=$ $1,168)$ had a spot urinary albumin:creatinine ratio (ACR) of $\geq 30 \mathrm{mg} / \mathrm{mmol}$. Three hundred forty-nine patients developed 
ESRD during a median observation period of 5.36 (interquartile range [IQR] 2.90-7.77) years, giving an incidence of 8.69 (95\% CI 7.78-9.60) per 1,000 personyears. Patients who developed ESRD were older (66, 57-72 vs 57, 46-67 years [median, IQR], $p<0.0001)$ and had a higher ACR (236.2, 70.7-441.0 vs 1.9, 0.7-8.9 mg/mmol [median, IQR], $p<0.0001)$, lower estimated GFR (47.6, $29.3-73.7$ vs $105.4,85.6-127.0 \mathrm{ml} \mathrm{min}^{-1} 1.73 \mathrm{~m}^{-2}$ [median, IQR], $p<0.0001)$ and packed cell volume $(0.359$, $0.324-0.359$ vs $0.407,0.379-0.437$ 1/1 [median, IQR], $p<$ $0.0001)$ than their counterparts who did not develop ESRD.

There were 749 deaths, 674 of which were not related to ESRD. Figure 1 shows the unadjusted and adjusted cumulative incidences of ESRD [3] to demonstrate the effect of competing risks as a result of non-ESRD-related deaths (adjusting for non-ESRD-related deaths). The unadjusted and adjusted cumulative incidences of ESRD were nearly identical within the initial 5 years of the observation period. From year 5 onwards, the adjusted incidence started to become lower than the unadjusted rate.

The dataset were evenly divided into the training dataset (186 events out of 3,821 patients) and the test dataset (163 events out of 3,695 patients). To examine the validity of the previous ESRD risk score [2], GFR (per $10 \mathrm{ml} \mathrm{min}$ $\left.1.73 \mathrm{~m}^{-2}\right), \log [\mathrm{ACR}]$ and packed cell volume (per $\left.0.1 \mathrm{l} / \mathrm{l}\right)$ were entered into the Cox proportional hazard model. However, GFR was no longer significant (hazard ratio $[\mathrm{HR}]=1.00,95 \%$ CI $0.99-1.01, p=0.9892)$. After removing GFR, $\log [\mathrm{ACR}](\mathrm{HR}=7.99,95 \%$ CI 6.24-10.22, $\beta=2.0776 \pm$ 0.1259 [SEM], $p<0.0001)$ and packed cell volume $(\mathrm{HR}=$ $0.34,95 \%$ CI $0.26-0.45, \beta=-1.0843 \pm 0.1400 \quad$ [SEM], $p<0.0001)$ remained significant. Thus, the modified ESRD risk score $=2.0776 \times \log [\mathrm{ACR}$ in $\mathrm{mg} / \mathrm{mmol}]-1.0843 \times$ packed cell volume per $0.1 \mathrm{l} / \mathrm{l}$. The 5 -year probability of $\mathrm{ESRD}=1-0.9937^{\mathrm{exp}(\text { the risk score }+3.2538)}$, where 0.9937 is the

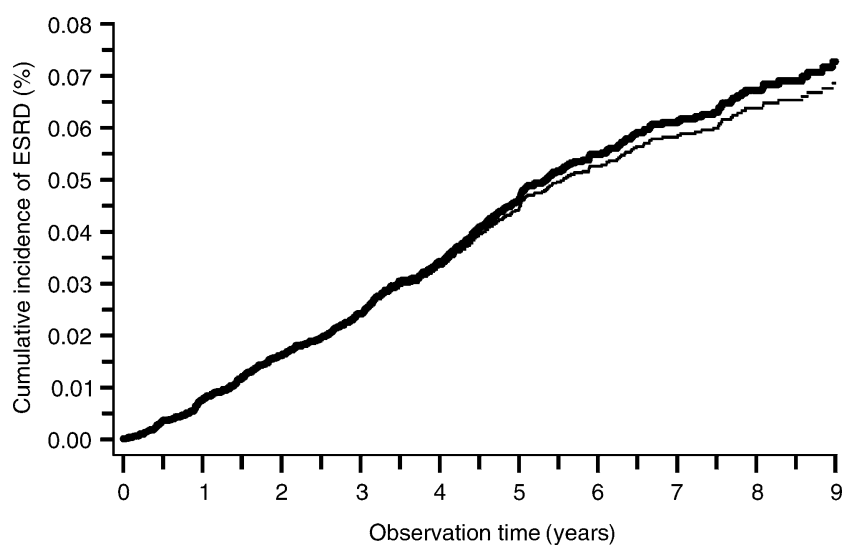

Fig. 1 Unadjusted (thick line) and competing risk-adjusted (thin line) cumulative incidence of ESRD in 7,516 Hong Kong Chinese patients with type 2 diabetes estimated 5-year survival function when the risk score is 3.2538 , the mean of the risk scores in the training dataset. The predicted rates and observed rates according to deciles of the risk score in the test dataset were not significantly different, as shown by the Hosmer-Lemeshow $\chi^{2}$ test $(p>$ $0.10)$. The unadjusted area under the receiver-operator characteristic curve (aROC) was 0.932 (95\% CI $0.908-$ 0.956), and the follow-up time- and censoring status-adjusted aROC [4] was 0.943 . At the cut-off point of greater than or equal to -0.8016 , the sensitivity and specificity were $86.1 \%$ and $90.3 \%$, respectively. The effects of other selected cut-off points of the risk score are listed in Electronic supplementary material Table 1.

In this repeat analysis, use of the C-MDRD equation [1] substantially reduced the ESRD incidence from 12.45 per 1,000 person-years [2] to 8.69 per 1,000 person-years in the present cohort. The predictive power of GFR was no longer significant and only packed cell volume and ACR remained significant. These findings suggest that low packed cell volume as a result of reduced GFR had greater predicting power than GFR in itself. Based on these findings, we hypothesise that, in populations with a low incidence of ESRD or a high incidence of anaemia owing to non-renal causes, GFR might have residual predictive power that cannot be accounted for by packed cell volume and ACR. After removal of GFR, the modified ESRD risk score maintained excellent discrimination. Since the impact of competing risk for ESRD, if any, was small over the initial 5 years of follow-up, the calculated 5-year ESRD probability by the modified equation was close to the real probability of ESRD. Hence, both the ESRD risk score and the probability equation can be used clinically if validated in other Chinese populations with type 2 diabetes. However, in light of the high risk of chronic kidney disease in Chinese individuals, the ESRD risk score may not be readily applicable to other type 2 diabetic populations with a low prevalence of renal complications. In addition, as the risk score was derived from a relatively affluent urban Chinese population, and given the diversity of lifestyles and disease patterns amongst Mainland Chinese individuals, recalibration of the absolute risk of ESRD might be needed for Chinese populations living in rural areas and in the northern part of China.

Duality of interest The study is partly supported by a Merck Sharp \& Dohme University Grant. R. R. Lyu and D. P. Yin are employees of Merck \& Co. C. S. Cockram and J. C. N. Chan are members of the Merck Worldwide Diabetes Advisory Board. J. C. N. Chan, P. C. Y. Tong, A. P. S. Kong, W. Y. So and C. C. Chow are sponsored by Merck Sharp \& Dohme. The other authors listed do not have any duality of interest. 


\section{References}

1. Ma YC, Zuo L, Chen JH et al (2006) Modified glomerular filtration rate estimating equation for Chinese patients with chronic kidney disease. J Am Soc Nephrol 17:2937-2944

2. Yang XL, So WY, Kong AP et al (2006) End-stage renal disease risk equations for Hong Kong Chinese patients with type 2 diabetes: Hong Kong Diabetes Registry. Diabetologia 49:22992308

3. Gooley TA, Leisenring W, Crowley J et al (1999) Estimation of failure probabilities in the presence of competing risks: new representations of old estimators. Stat Med 18:695-706

4. Chambless LE, Diao G (2006) Estimation of time-dependent area under the ROC curve for long-term risk prediction. Stat Med 25:3474-3486 